\title{
Learning by doing and learning by thinking: an fMRI study of combining motor and mental training
}

\author{
C.-J. Olsson ${ }^{1, *}$, Bert Jonsson ${ }^{2}$ and Lars Nyberg ${ }^{1,3,4}$ \\ 1. Department of Integrative Medical Biology (Physiology), Umeå University, Umeå, Sweden \\ 2. Department of Psychology, Umeå University, Umeå, Sweden \\ 3. Department of Radiation Sciences, Umeå University, Umeå, Sweden \\ 4. Umeå Center for Functional Brain Imaging (UFBI), Umeå, Sweden \\ Edited by: Kenneth Hugdahl, University of Bergen, Norway \\ Reviewed by: Alfons 0 . Hamm, Arndt University of Greifswald, Germany \\ Jarl Risberg, University of Lund, Sweden
}

\begin{abstract}
The current study investigated behavioral and neural effects of motor, mental, and combined motor and mental training on a finger tapping task. The motor or mental training groups trained on a finger-sequence for a total of 72 min over 6 weeks. The motor and mental training group received 72 min motor training and in addition $72 \mathrm{~min}$ mental training. Results showed that all groups increased their tapping performance significantly on the trained sequence. After training functional magnetic resonance imaging (fMRI) data was collected and indicated training specific increases in ventral pre-motor cortex following motor training, and in fusiform gyrus following mental training. Combined motor and mental training activated both the motor and the visual regions. In addition, motor and mental training showed a significant increase in tapping performance on an untrained sequence (transfer). fMRI scanning indicated that the transfer effect involved the cerebellum. Conclusions were that combined motor and mental training recruited both motor and visual systems, and that combined motor and mental training improves motor flexibility via connections from both motor and cognitive systems to the cerebellum.
\end{abstract}

Keywords: transfer, fMRI, cerebellum, mental, motor, training

\section{INTRODUCTION}

Over the year's considerable amount of research has been examining mechanisms underlying motor training. It is clear that motor training is associated with changes of neural activation in primary motor cortex (Karni et al., 1995), SMA (Picard and Strick, 2001; Tanji, 2001), and cerebellum (Debaere et al., 2004; Hikosaka et al., 1998; Middleton and Strick, 2000). However, motor training is not the only strategy one can use to improve motor skills. Studies have convincingly shown that mental training using motor imagery can result in positive effects on motor abilities (Driskell et al., 1994; Grouios, 1992; Mamassis and Doganis, 2004; Peynircioglu et al., 2000).

When comparing motor execution and mental imagery, research has shown that there is a functional equivalence between the two methods. For example, studies have shown that when increasing the walking distance between two points the actual walking time as well as the mental walking time increases (Decety and Jeannerod, 1995; Jeannerod, 2001). Likewise, neuroimaging studies, using, e.g., functional magnetic resonance imaging (fMRI),

*Correspondence: C.-J. Olsson, Department of Integrative Medical Biology (Physiology), Umeå University, S-901 87 Umeå, Sweden. e-mail: cj.olsson@physiol.umu.se

Received: 28 April 2008; paper pending published: 15 May 2008; accepted: 04 June 2008; published online: 18 June 2008.

Citation: Front. Hum. Neurosci. (2008) 2: 5. doi: 10.3389/neuro.09.005.2008

Copyright (c) 2008 C.-J. Olsson, Bert Jonsson and Lars Nyberg. This is an open-access article subject to an exclusive license agreement between the authors and the Frontiers Research Foundation, which permits unrestricted use, distribution, and reproduction in any medium, provided the original authors and source are credited. have shown that motor imagery and motor execution share a functional neural activation pattern, i.e., executing and imagining a motor task activate similar neural networks (Jeannerod, 1995; Kosslyn et al., 2007; Lacourse et al., 2005). However, recent neuroimaging data indicate that the underlying neural mechanisms from practicing using motor imagery are partly different from those after actual motor practice, with motor practice more associated with increases in SMA and cerebellum and mental training more associated with visual regions (Nyberg et al., 2006).

Little is known about the effects on motor performance and neural activity of combining motor and mental training. This is so despite the fact that a combination of motor and mental training is what typically would be used in athletic contexts, and it is fair to say that a widely held assumption is that a combination of motor and mental training will facilitate performance. This assumption, however, rests on the idea of functional equivalence. Based on their findings of different neural correlates of motor and mental training, Nyberg et al. (2006) have suggested that combined motor and mental training might result in interference effects. However, studies of combined motor and mental training have shown that it has positive effects on motor performance (Brouziyne and Molinaro, 2005; Feltz and Landers, 1983; Pie and Tenenbaum, 1996). Also, even though the use of two different neural systems may result in interference, extended practice during interference has positive long-term effects on motor performance (Imamizu et al., 2007). Thus, the assumption of having positive effects after combining motor and mental training is underpinned by findings from several studies. 
The present study was modeled after the previous study by Nyberg et al. (2006), and examined how more extended (over 5 weeks) motor or mental training affects performance on a sequential finger tapping task and the underlying neural networks measured by fMRI. Specifically, participants were first tested on two different finger-tapping sequences, then practiced for a period using either motor, mental or combined motor and mental training on one of the two sequences, and were finally tested again on both sequences (cf., Karni et al., 1995). This design allowed us to evaluate any differences in performance and neural activity following the different training methods. Furthermore, we could examine possible transfer effects to the untrained sequence concerning both performance and neural activity.

We hypothesized that motor and mental training would result in a performance increase with a greater increase for motor training, and that extended motor or mental training would result in increased activation in motor regions (e.g., SMA and cerebellum) and non-motor, visual regions, respectively (cf., Nyberg et al., 2006). When combining motor and mental training, we hypothesized that the training effect would be based on similar neural networks as seen after motor and mental training in isolation, possibly leading to an additive effect on tapping performance. An alternative hypothesis was that combined training would not boost performance beyond the level of motor training only, and possibly even lead to reduced performance compared to motor or mental practice due to interference effects. Such interference effects might be translated into lowered training-related signals in the relevant motor and visual regions.

\section{MATERIALS AND METHODS PARTICIPANTS}

Thirty, neurologically healthy, right handed (by self report), subjects participated on a voluntary basis. There were 17 males, and 13 females (mean age 20.3 years). They were randomly assigned to one of three training groups, with 10 subjects per group. Six participants from each group took part in the fMRI scanning session. The remaining 12 participants only participated in the non-scanning part of the study. The participants had given their written informed consent and the ethical committee at the University Hospital of Northern Sweden approved the study. For taking part of this study, the participants were given a monetary reward $(\$ 100)$.

\section{PROCEDURE}

The overall protocol for each of the three training groups (motor, mental, and combined motor and mental) was pre-test, followed by training, and finally post-test.

During pre-test, the participants were instructed that each of the four fingers of the left hand represented a single digit number, where the index finger was number one and little finger was number four. They were then told to, as accurate and as fast as possible, sequentially tap the fingers against a laptop keyboard using F G H J according to a sequence of digits presented on a computer screen placed in front of them. They were asked to continue the tapping for as long as the sequence appeared on the screen. The hand was covered by a cardboard box. Two different sequences were used based on previous research (e.g., Karni et al., 1995; Nyberg et al., 2006): A = 23142 and B = 24 432 . The first sequence was presented for $30 \mathrm{~s}$, followed by $30 \mathrm{~s}$ rest, and thereafter the second sequence. During the resting periods the display showed five $\mathrm{x}$ 's $(\mathrm{x} \times \mathrm{x} \times \mathrm{x})$ and the participants were instructed to rest. This test protocol was performed three times. The tapped sequences were recorded using E-Prime 1.1 (Psychology Software Tools, PA, USA).

During the training sessions, each of the participants trained on one of the two sequences (A or B). The assignment of training sequence $\mathrm{A}$ or $\mathrm{B}$ was counterbalanced across subjects. The sequence was presented on a computer screen and the participants trained under the inspection of the experimenter. The non-trained sequence only appeared during the pre- and posttest in order to evaluate possible transfer effects. The motor training group tapped the sequence on a table-top with their hand covered by a cardboard box. The mental training group was instructed to use a first person (internal) imagery perspective where it was emphasized that it was important to "feel" as if the sequence was executed without actually moving the fingers. At each training session the participants mentally tapped the sequence as accurate and as fast as possible and they were encouraged to increase the speed as much as possible. In order to prevent finger movements they held their hands clasped together visible on the table top. Sessions of motor only or mental only training were carried out twice a week for 6 weeks. In each training session the participants performed the allotted sequence during four $90 \mathrm{~s}$ periods with $60 \mathrm{~s}$ rest periods in between. Thus, the total training time for these groups was $72 \mathrm{~min}$ over a period of 6 weeks. On each training session, the group with combined motor and mental training first did the same training as the motor group and then the same training as the mental group. Thus the total training time for the combined training group was 144 min over 6 weeks.

Following 1 or 2 days after the last training session, all participants were tested on both sequences to assess training and transfer effects. The post-testing was done according to the same protocol as at pre-test.

\section{fMRI METHODS}

When the training phase was completed, the participants underwent fMRI-scanning. In the fMRI-scanner, the participants were randomly presented one of the two sequences. Every time a sequence appeared on a semi-transparent screen at the end of the bore of the scanner they were instructed to as fast and accurately as possible tap the sequence once. A sequence was displayed for $2.5 \mathrm{~s}$ immediately followed by the next sequence. Each sequence was presented 30 times. The participants executed the task with a four-button response pad (Lumi-touch reply system, Lightwave Medical Industries, Canada). The number of tapped sequences was registered using a laptop computer running E-Prime 1.1 (Psychology Software Tools, PA, USA). Cushions inside the head coil reduced head motion and the participants wore headphones to reduce noise from the scanner.

For fMRI-scanning a Philips Intera $1.5 \mathrm{~T}$ system (Philips Medical Systems, Netherlands) was used. Blood-oxygen-leveldependent (BOLD) $\mathrm{T} 2{ }^{\star}$ weighted images were collected using a gradient echo-planar imaging (EPI) sequence. The following imaging parameters were used: echo time: $50 \mathrm{~ms}$, repetition time: $3000 \mathrm{~ms}$, flip angle: $90^{\circ}$, field of view $22 \mathrm{~cm} \times 22 \mathrm{~cm}$, matrix size: $64 \times 64$ and slice thickness: $4.4 \mathrm{~mm}$.

\section{STATISTICAL ANALYSIS \\ Behavioral data}

Each button press made by the participants was counted as well as all correctly completed sequences for both the pre-test and the post-test. An ANOVA was made on the pre-test results to 
make sure that the participants in the three groups were at an equal level before training. In order to evaluate training related changes on performance for each training method, respectively, planned comparisons with paired $t$-tests were used. Further, in order to evaluate between group differences, the increase in tapping performance (i.e., the total number of correctly completed sequences) was first calculated taking the post-results minus the pre-results. The increase in tapping performance was then analyzed using ANOVA. Separate analyzes were made for the untrained and for the trained sequence.

The number of correctly tapped sequences was also counted from the fMRI sessions. However an effect of the event related design used when collecting brain data was that it changed how we were able to compare the different training methods behaviorally in the scanner. Instead of comparing the total number of completed sequences during a fixed time period, as outside the scanner, we were now only able to look at the number of correctly tapped sequences out of a fixed number of sequences presented (30). Consequently, the scale when presenting the data will be different and it may also create ceiling effects. Due to these reasons we consider the performance measures collected outside the scanner to be of greater interest. A second behavioral measure from the fMRI session was the average total time to complete the sequences (i.e., mean time per sequence). These measures were also analyzed using ANOVAs. All statistical tests were performed at a $p<0.05$ level of significance. Post hoc testing (least significant difference) was used to specify group differences after significant ANOVAs.

\section{Neural data}

To convert the fMRI images to Analyze format, the MRIcro program was used (Rorden and Brett, 2000). Pre-processing was done with SPM2 (Wellcome Department of Cognitive Neurology, London, UK) and included slice timing, realignment, unwarping, normalization to an EPI template of the Montreal Neurological Institute (MNI) standard brain, and smoothed using a Gaussian kernel with a FWHM of $8 \mathrm{~mm}$. The general linear model was used to set up single subject statistical analyses. Statistical parametric maps (SPMs) were then generated using $t$-statistics. To reveal differences in neural activity between the different training methods, and between the trained and the untrained sequence, the total time for each correctly tapped sequence was extracted and used to define a small block. A resting period was calculated as the time from the participant's last button press for one sequence until the next sequence appeared on the screen. The trained and the untrained sequences as well as the rest period (i.e., the period in between sequences) were defined as separate regressors.

We were primarily interested in finding regions associated with effects of motor training and mental training, respectively. We considered a region responding specifically to motor training as a brain area that showed stronger activation when reproducing the trained sequence as compared to the untrained sequences for the groups that had only motor training (i.e., the motor group and the group with combined motor and mental training, $n=12$ ). Conversely, brain regions specifically associated with mental training should show a stronger activation for the group that trained mentally and for the group that received combined motor and mental training $(n=12)$. The contrasts (trained-untrained for motor and mental training, respectively) were carried out at a statistical threshold of 0.005 uncorrected. Random-effects analyses were performed to reveal group-averaged data.
Thereafter, BOLD-values were extracted for the local maxima within identified regions for the trained and the untrained sequence. A positive difference between the BOLD-values for trained-untrained sequence would indicate a training-specific region for either motor training or mental training, and a negative or zero difference would indicted that this regions was not training-specific. ANOVAs were then used to see whether these differences were significantly different between groups and therefore could be associated with mental or motor training, respectively. Correlational analyses were computed for training specific regions (beta-values) after both motor and mental training with tapping performance during scanning.

For visualization of cerebellar regions, a cerebellar anatomical image from Caret (http://brainmap.wustl.edu/caret) was used. For cerebral images, MRIcro (www.sph.sc.edu/comd/ rorden/mricro.html) was used and BOLD-plots were visualized using in-house developed software (DataZ). The BOLD-plots were calculated by dividing the actual BOLD-value for the specific peaks of each participant with the mean BOLD-value of the session for the same participant.

Pending a significant performance increase on the untrained sequence (transfer), the contrast (untrained-rest) was conducted for the specific group(s) using a statistical threshold of 0.005 uncorrected, followed by BOLD-plots for each group of the peak value from the observed clusters. All coordinates describing peak-activations are in MNI-space.

\section{RESULTS}

Figure 1 summarizes the behavioral data acquired outside the scanner as well as during fMRI-scanning.

\section{BEHAVIORAL DATA \\ Pre-scanning session}

The participants conducted on average 20.5 (SD 10.3) correctly tapped sequences before the start of the training. The accuracy was relatively high, $79 \%$ of the attempted sequences were correct. The performance levels before training did not differ significantly among groups $F_{2,27}=2.4, p>0.05$. Thus, possible differences between the groups after training should reflect the different training methods.

Planned comparisons indicated that all groups increased their tapping performance significantly after training; $t_{9}=6.0$ (motor training), $t_{9}=4.2$ (mental training), and $t_{9}=8.5$ (combined training) all $p$-values $<0.05$. Accuracy increased to $88 \%$. Furthermore, the tapping-performance increase was significantly different across groups $F_{2,27}=4.4, p<0.05$, (Figure 1A). Combined motor and mental training as well as motor training only resulted in a greater increase in performance than mental training $(p<0.05$ for both post hoc comparisons), while there was no significant difference between combined training and motor training only.

For the untrained sequence, planned comparison showed that there was a significant increase after the training period (transfer) for the combined motor and mental group $t_{9}=5.3$, $p<0.05$ whereas no transfer was observed for either motor training $t_{9}=0.8, p>0.05$ or for mental training $t_{9}=1.2, p>0.05$. Moreover, a significant group difference for the increase in tapping performance was found $F_{2,27}=5.5, p<0.05$. Subsequent post hoc testing revealed that the increase in tapping performance for the combined motor and mental training group was significantly greater relative to motor training as well as to mental training, (both $p$-values $<0.05$ ). The accuracy was similar as before training, $78 \%$. 

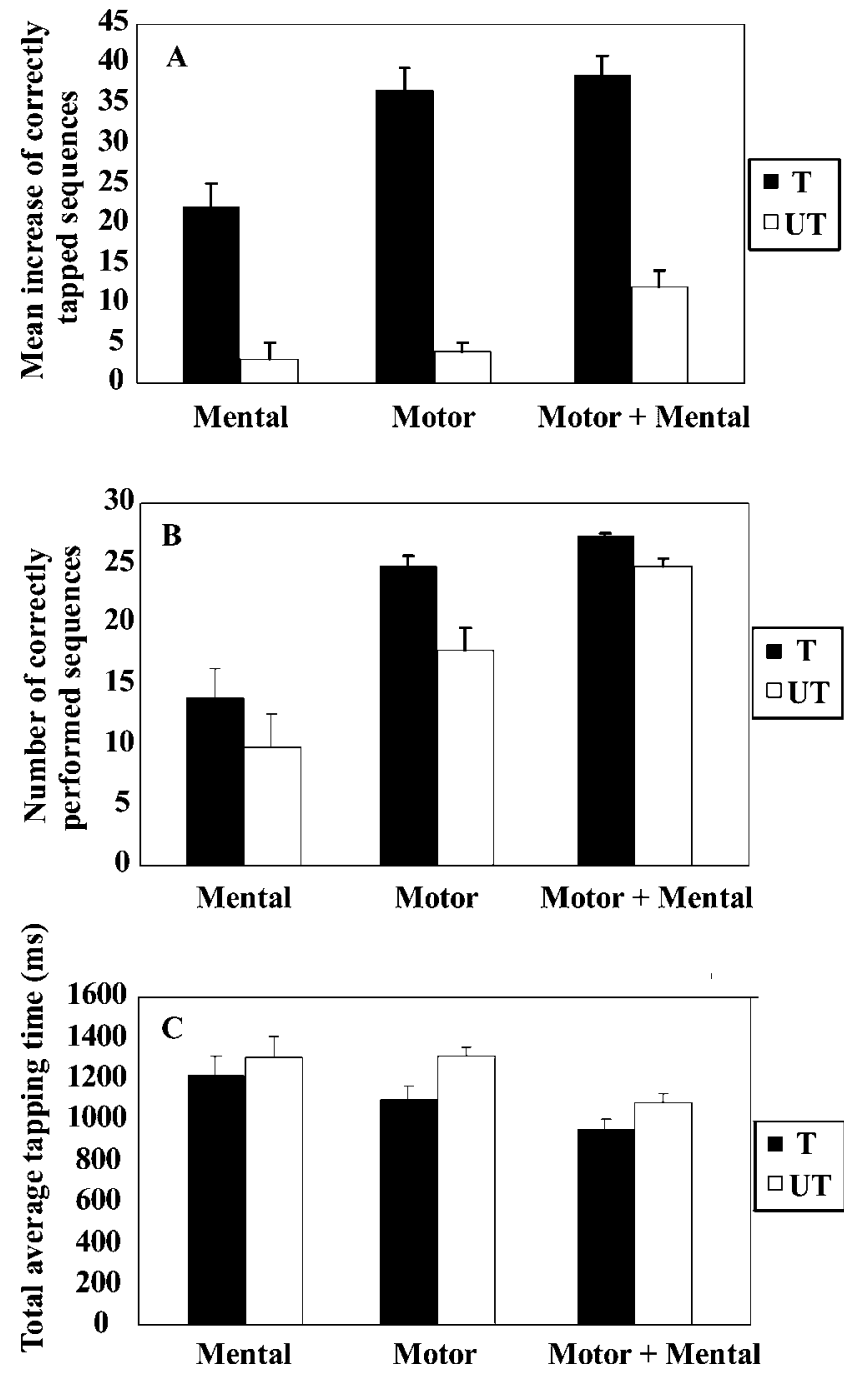

Figure 1 | Behavioral performances following motor, mental, or combined motor and mental training. All measures revealed a similar pattern, where combined motor and mental training and motor training only were more effective compared to mental training. There was a non-significant trend towards an additive effect of combining motor and mental training. Moreover, combining motor and mental training resulted in performance increase on the untrained sequence (transfer). Data for the trained ( $T$ ) and the untrained (UT) sequences are presented for $(\boldsymbol{A})$ the increase in tapping performance outside the scanner, $(\boldsymbol{B})$ the mean number of correctly tapped sequence during fMRIscanning, and $(\boldsymbol{C})$ the average tapping time during the fMRI-scanning. The bars denote standard error.

\section{Control analysis - magnitude versus type of training}

The finding of a significant transfer effect in the group with combined training but not in the groups with only mental or motor training could potentially result from a greater time spent on training given that the participants in the combined group performed both training protocols. To test whether the transfer effect was due to the amount of training, rather than the combination of training protocols, a fourth group of participants $(n=6)$ was added as a control group. This group trained the same amount (144 min) and according to the same procedure using motor training only. Before the start of training (baseline performance), the average amount of correctly tapped sequences was 29.2. After training, the group on average increased their performance on the trained sequence with 40.8 correctly performed sequences, and their performance on the untrained sequence with 6.8 correctly performed sequences. A significant performance increase was revealed for the trained sequence $t_{5}=3.3, p<0.05$ but not for the untrained sequence $t_{5}=1.9, p>0.05$, suggesting that no reliable transfer occurred. Thus, the transfer effect for the combined group was unlikely a result of higher training load per se.

\section{Behavioral data recorded in the scanner}

The tapping performance for the trained sequence differed among groups, $F_{2,15}=5.3, p<0.05$, (Figure 1B). Combined motor and mental training differed significantly from mental training $(p<0.05$; post hoc), and there was a tendency toward a significant difference between the motor and mental groups $(p=0.07)$. From the tapping time recordings of how fast each participant performed the sequences, a similar graded pattern emerged (Figure 1C). That is, the group with combined motor and mental training performed fastest, followed by the motor training group and finally the mental training group. However, these differences did not reach statistical significance, $F_{2,15}=1.0$, $p>0.05$. For the untrained sequence there was a statistically significant difference among groups in the number of correctly tapped sequences, $F_{2,15}=3.6, p=0.05$. Post hoc testing indicated that the motor and mental group performed better compared to the mental training group, $p<0.05$, thus replicating the effect observed outside the scanner. For the average tapping time no significant group differences were found, $F_{2,15}=1.25, p>0.05$.

Taken together, the behavioral data showed that both motor and mental training enhanced tapping performance, but motor training led to a greater facilitation of performance. Moreover, the combination of motor and mental training led to more and faster performed sequences, but this trend was not statistically significant. Finally, a significant transfer effect to the untrained sequence was only seen for the combined motor and mental training group.

\section{NEURAL DATA}

Figure 2 shows activation changes associated with reproducing the trained sequence following motor training and mental training. A training-specific activation increase was found in ventral pre-motor cortex (BA 6/44), $(x, y, z=50,12,16)$ following motor training, and in fusiform gyrus (BA 19), $(x, y, z=-30$, $-76,-12)$ following mental training. ANOVAs on the BOLD signal change revealed that in the fusiform gyrus there was significantly greater activation for the mental training group and the combined motor and mental training group compared to the motor training group. In ventral pre-motor cortex there was significantly greater activation for the motor training group and the combined motor and mental training group compared to the mental training group $\left(F_{1,16}=7.5\right.$, and $F_{1,16}=8.1$, respectively, all $p$-values $<0.05)$. The correlation between tapping performance on the trained sequence for participants training motorically (motor only or combined, $n=12$ ) and the BOLD-value in pre-motor cortex was significant, $r=0.608, p<0.05$ (Figure 3 ). For mental training a similar correlation between tapping performance and BOLD-activation in fusiform gyrus did not reach statistical significance, $r=0.262, p>0.05$.

The behavioral data showed that only combined motor and mental training resulted in a significant transfer effect. A region, 88 voxels, in left (ipsilateral) cerebellum was associated with this selective transfer effect (Figure 4). The peak activation in this region was in the posterior lobe $[(x, y, z=-18,-70,-12)$, $T=8.49]$. A second peak was found more anterior in the same 


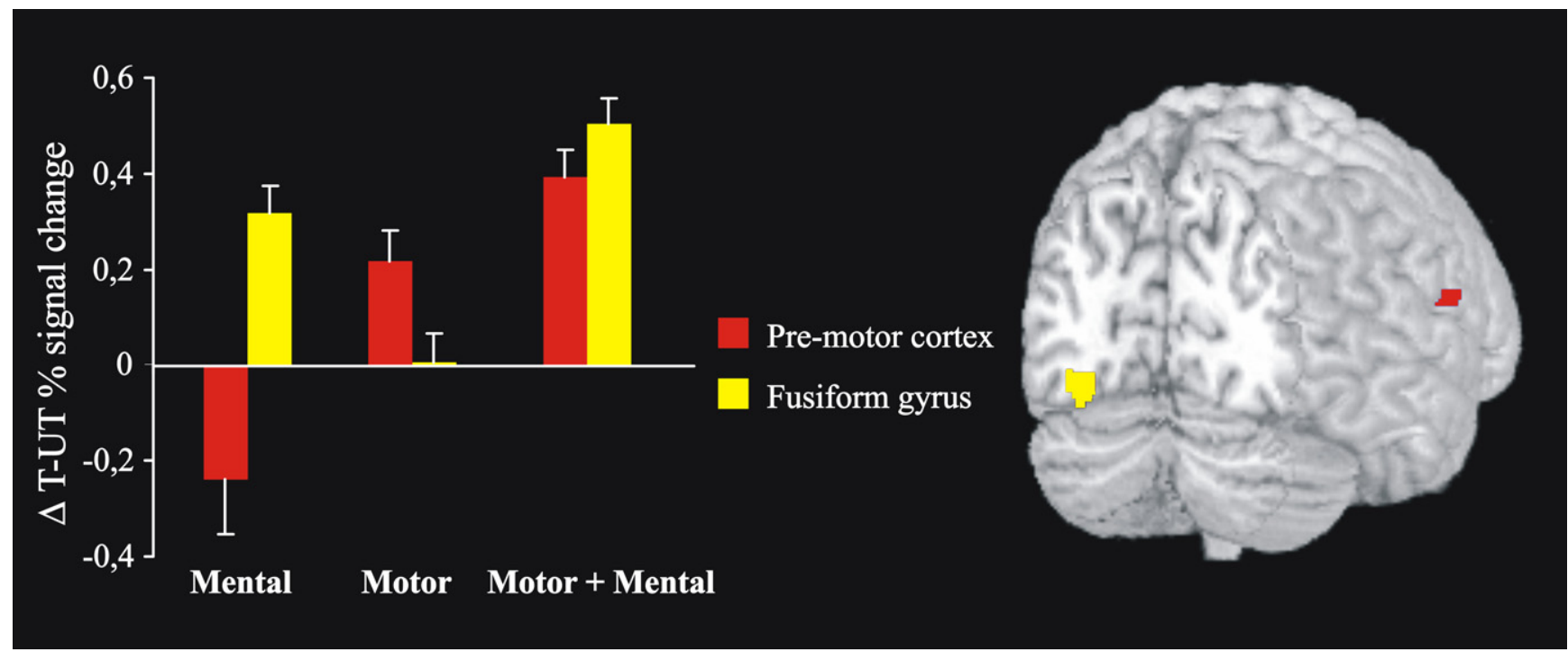

Figure 2 | After 6 weeks of motor training, a significant activation increase was observed in the contralateral (right) ventral pre-motor cortex (red) during performance of the trained sequence. After mental training, activation was significantly increased in the left fusiform gyrus (yellow). Both these regions showed increased activity after a combination of motor and mental training. Bars indicate differences in BOLD signal change between the trained and untrained sequences $(\Delta \mathrm{T}-\mathrm{UT})$ and error bars are standard error.

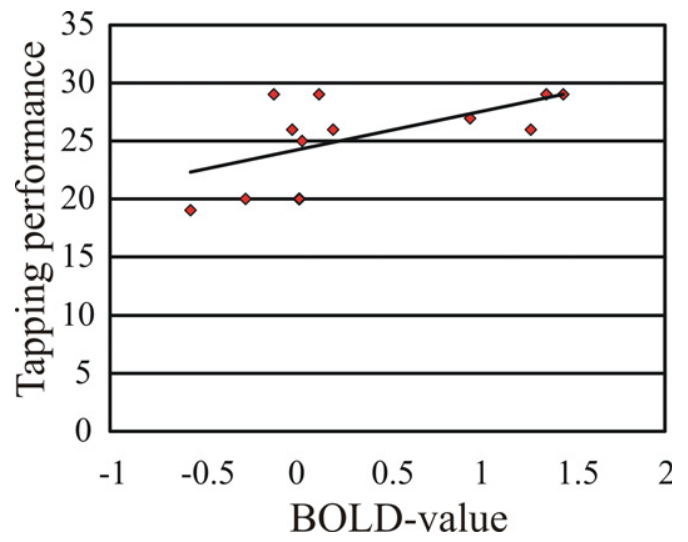

Figure 3 | The correlation between tapping performance inside the scanner on the trained sequence and the BOLD signal change in pre-motor cortex (beta-value) was significant.

lobe $[(x, y, z=-16,-60,-14), T=6.32]$. Analyses of the BOLDvalues from the strongest peak revealed a significant difference among groups $F_{2,15}=11.9$. The combined motor and mental group had a significantly stronger activation compared to motor only and mental only training groups (both $p$-values $<0.05$; post hoc).

\section{DISCUSSION}

\section{TRAINING SPECIFIC EFFECTS}

We have shown that both motor and mental training improved task performance, with motor training being more effective than mental training. This is in accordance with many other studies (e.g., Driskell et al., 1994). Furthermore, we have also shown that the underlying neural mechanisms of motor and mental training differ (cf., Nyberg et al., 2006). When participants after training performed the trained sequence, a region of the motor system showed more activation following motor training compared to mental training. It was located in ventral pre-motor cortex
(BA 6/44) slightly inferior to the arm and hand area (Dechent and Frahm, 2003; Dum and Strick, 2002; Yousry et al., 1997), indicating a role in organizing the trained motor sequence (Sergent et al., 1992). Further support was obtained from the correlational analysis, showing a significant correlation between tapping performance and pre-motor activity. Mental training was associated with greater activation compared to motor training in an extrastriate region of the ventral pathway, located in the fusiform gyrus (BA 19) of the visual cortex. Previous research has related regions of the visual system to mental training of finger tapping sequences (Nyberg et al., 2006). Extrastriate regions of the visual cortex have been associated with the transformation of visual information into complex movements during imagery of music performance (Meister et al., 2004) and when reading musical scores (Sergent et al., 1992). Thus, it is likely that imagining the finger sequences during the mental training created a visual memory of the sequence that is stored in this region and reactivated during actual task performance.

In addition to examining the effects following motor or mental training on the finger tapping task, we investigated the effects, on the same finger tapping task, of combining mental training and motor training. Combined training led to a slight increase in tapping performance relative to motor only training, which argues against an interfering effect of combined training, but the level of increase was non-significant which precludes strong conclusions regarding additive effects. However, here it should be noted that doubling the amount of motor training (the control experiment) did not show significant additive effects either. One possible reason may be functional ceiling effects, such that the magnitude of improvement on the finger tapping task has a limit. A second reason may be that in order to result in further increased effects, the participants must engage in deliberate practice that is designed to optimize the performance and not just add more training hours or a different training method (Ericsson et al., 1993; Keith and Ericsson, 2007).

Importantly, for combined motor and mental training both regions associated with motor training and with mental training 

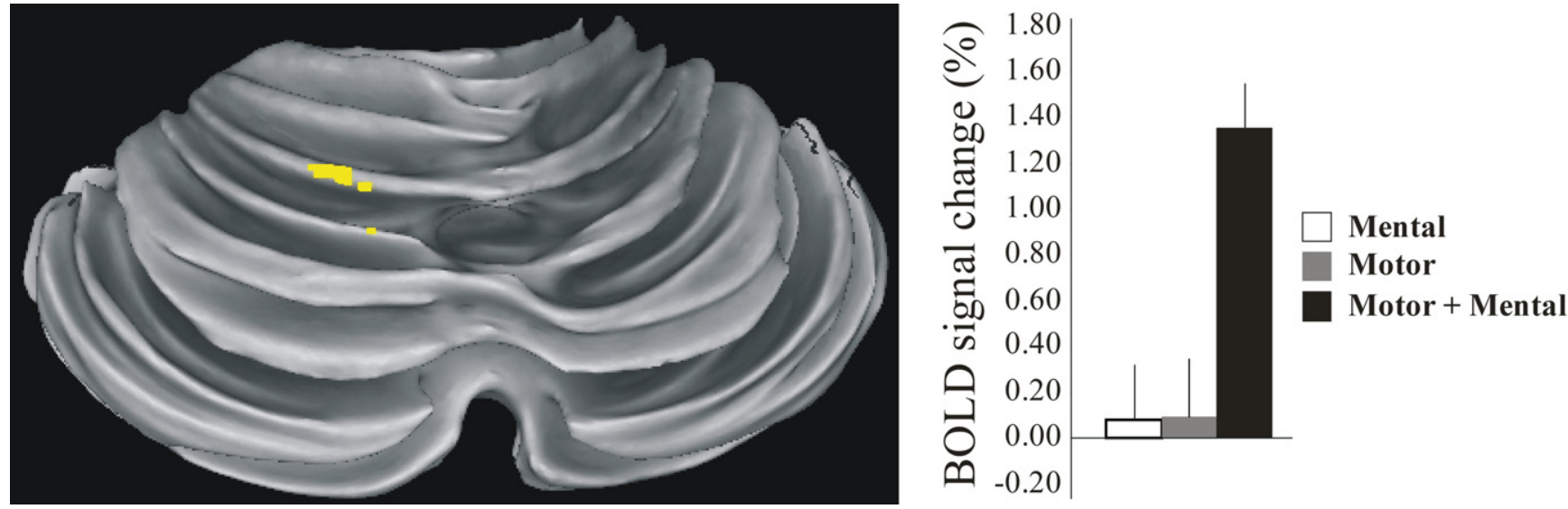

Figure 4 | Increased activity in posterior ipsilateral (left) cerebellum was observed during performance of the untrained sequence (transfer) after combined motor and mental training but not after motor only or mental only training. Bars indicate the BOLD signal change in relation to the mean BOLDvalue over the session, with error bars denoting standard errors.

were activated during task performance. Thus, after combining motor and mental training, components of both the motor and the visual system were activated. This suggests that after using both motor and mental training methods the trained sequence is represented in partly distinct brain regions. Future studies should focus on explaining what effects this extra representation in the visual system might have on motor performance in more complex and, hence, more cognitively demanding tasks such as in athletic contexts. It is also important to consider whether it is the motor training that supports the mental training or if it is the other way around. Previous research suggests that mental imagery and associated brain activity differ depending on the nature of the underlying motor representations (Olsson et al., 2008). Thus, it remains a possibility that, as in this study, having mental training follow motor training contributed to the observed result. At the same time, finger movement representations should have been well developed in all participants, suggesting that the joint activation of visual and motor regions may be independent of the ordering of the training methods.

\section{COMBINED MOTOR AND MENTAL TRAINING FACILITATES TRANSFER}

An unexpected effect from the combined motor and mental training was the improvement on the untrained sequence. The control experiment suggested that this effect did not result from an increased amount of training. Also, since there was no transfer for any of the other groups, the transfer was likely not an effect of similarities between the two sequences. Traditionally, it has been assumed that what you learn is specific both to the context and to the task (Seidler, 2004), which would explain why motor or mental training only showed training-related improvements on the trained sequence and not on the untrained sequence. In contrast, Wohldmann et al. (2007) suggested that mental training alone results in transfer. The present findings indicate that motor training is necessary in order to first establish a motor representation and a general tapping skill. Imagery training can then be used to learn how to incorporate visual stimuli and associate them with a motor output. Consequently, because of combined motor and mental training, the motor flexibility will increase and the participants will be able to decompose a new sequence into the numbers associated with each finger and then perform in the order specified by the new sequence.
The neural data indicated that the ipsilateral posterior cerebellar hemisphere was involved in the transfer. This region, between lobules VI and VII, has previously been associated with plastic changes of internal models (Diedrichsen et al., 2005). Studies have suggested that the cerebellum generates an internal model used to learn sensorimotor associations as a basis for feedforward motor control (Fujita, 2006; Wolpert et al., 1995), and that the cerebellum is responsible for selecting the proper motor behavior and optimize performance (Gonzalez et al., 2005; Penhune and Doyon, 2005). We suggest that the connections between cognitive parts of cerebral cortex and lateral cerebellum (Thach, 2007) and between primary motor cortex and the cerebellum (Kelly and Strick, 2003) enhance the motor flexibility when combining motor and mental training. When engaging in motor training, the motor program, components of which may be stored in the cerebellum (Attwell et al., 2002; Jenkins et al., 1994; Medina et al., 2001), is strengthened. Mental training also engages the cerebellum (Gerardin et al., 2000; Lacourse et al., 2004; Luft et al., 1998; Naito et al., 2002). Likely, however, mental training results in a more abstract representation compared to motor training. Hypothetically, the combination of motor and mental training improves the transformation of visual information into motor execution as well as the motor program in itself, hence improving the ability for the cerebellum to select the proper motor behavior on basis of stored motor programs (Obayashi, 2004). The fact that increased cerebellar activation was not associated with the trained sequence in either group indicates that the transfer effect was not reflecting enhanced behavioral performance. Instead, we propose that increased motor flexibility follows from adding mental training to motor training which makes it possible for the cerebellum to integrate the visual representation from the mental training with the motor representation from the motor training.

\section{CONCLUSION}

We conclude that motor and mental training are associated with functional changes in partly distinct regions (motor versus visual) of the brain. Although the combination of motor and mental training engages both regions, this did not strongly affect task performance. However, applying both motor and mental 
training methods improved motor flexibility, as reflected by transfer to an untrained sequence in our experiment. That adding mental training to motor training turns out to be more effective than motor training only may be of relevance for athletes and in rehabilitation programs.

\section{CONFLICT OF INTEREST STATEMENT}

The authors declare that the research was conducted in the absence of any commercial or financial relationships that could be construed as a potential conflict of interest.

\section{ACKNOWLEDGEMENTS}

This study was supported by a grant to Lars Nyberg from Centrum för Idrottsforskning (CIF). We thank Roland S. Johansson for comments on an earlier draft of this manuscript. We would also like to thank Micael Andersson for statistical support, Anne Larsson for helping collecting the functional data, and the participants for taking part in this study.

\section{REFERENCES}

Attwell, P. J. E., Cooke, S. F., and Yeo, C. H. (2002). Cerebellar function in consolidation of a motor memory. Neuron 34, 1011-1020.

Brouziyne, M., and Molinaro, C. (2005). Mental imagery combined with physical practice of approach shots for golf beginners. Percept. Mot. Skills 101 203-211.

Debaere, F., Wenderoth, N., Sunaert, S., Van Hecke, P., and Swinnen, S. P. (2004) Changes in brain activation during the acquisition of a new bimanual coordination task. Neuropsychologia 42, 855-867.

Decety, J., and Jeannerod, M. (1995). Mentally simulated movements in virtual reality: does Fitts's law hold in motor imagery? Behav. Brain Res. 72 127-134.

Dechent, P., and Frahm, J. (2003). Functional somatotopy of finger representations in human primary motor cortex. Hum. Brain Mapp. 18, 272-283.

Diedrichsen, J., Hashambhoy, Y., Rane, T., and Shadmehr, R. (2005). Neural correlates of reach errors. J. Neurosci. 25, 9919-9931.

Driskell, J. E., Copper, C., and Moran, A. (1994). Does mental practice enhance performance? J. Appl. Psychol. 79, 481-492.

Dum, R. P., and Strick, P. L. (2002). Motor areas in the frontal lobe of the primate. Physiol. Behav. 77, 677-682.

Ericsson, K. A., Krampe, R. T., and Teschromer, C. (1993). The role of deliberate practice in the acquisition of expert performance. Psychol. Rev. 100, 363-406.

Feltz, D. L., and Landers, D. M. (1983). The effects of mental practice on motor skill learning and performance: a meta-analysis. J. Sport Psychol. 5, 25-57.

Fujita, M. (2006). Feed-forward associative motor learning by the cerebellum. Cerebellum 5, 298-299.

Gerardin, E., Sirigu, A., Lehericy, S., Poline, J.-B., Gaymard, B., Marsault, C., Agid, Y., and Le Bihan, D. (2000). Partially overlapping neural networks for real and imagined hand movements. Cereb. Cortex 10, 1093-1104.

Gonzalez, B., Rodriguez, M., Ramirez, C., and Sabate, M. (2005). Disturbance of motor imagery after cerebellar stroke. Behav. Neurosci. 119, 622-626.

Grouios, G. (1992). The effect of mental practice on diving performance. Int. J. Sport Psychol. 23, 60-69.

Hikosaka, O., Miyashita, K., Miyachi, S., Sakai, K., and Lu, X. (1998). Differential roles of the frontal cortex, basal ganglia, and cerebellum in visuomotor sequence learning. Neurobiol. Learn. Mem. 70, 137-149.

Imamizu, H., Sugimoto, N., Osu, R., Tsutsui, K., Sugiyama, K., Wada, Y., and Kawato, M. (2007). Explicit contextual information selectively contributes to predictive switching of internal models. Exp. Brain Res. 181, 395-408.

Jeannerod, M. (1995). Mental imagery in the motor context. Neuropsychologia $33,1419-1432$

Jeannerod, M. (2001). Neural simulation of action: a unifying mechanism for motor cognition. Neuroimage 14, S103-S109.

Jenkins, I. H., Brooks, D. J., Nixon, P. D., Frackowiak, R. S., and Passingham, R. E. (1994). Motor sequence learning: a study with positron emission tomography. J. Neurosci. 14, 3775-3790.
Karni, A., Meyer, G., Jezzard, P., Adams, M. M., Turner, R., and Ungerleider, L. G. (1995). Functional MRI evidence for adult motor cortex plasticity during motor skill learning. Nature 377, 155-158.

Keith, N., and Ericsson, K. A. (2007). A deliberate practice account of typing proficiency in everyday typists. J. Exp. Psychol. Appl. 13, 135-145.

Kelly, R. M., and Strick, P. L. (2003). Cerebellar loops with motor cortex and prefrontal cortex of a nonhuman primate. J. Neurosci. 23, 8432-8444.

Kosslyn, S. M., Ganis, G., and Thompson, W. L. (2007). Mental imagery and the human brain. In: Progress in Psychological Science Around the World, Vol. 1, Neural, Cognitive and Developmental Issues, Q. Jing, M. R. Rosenweig, G. d'Ydewalle, H. Zhang, H.-C. Chen and K. Zhang, eds (New York, NY, Psychology Press), pp. 195-209.

Lacourse, M. G., Orr, E. L. R., Cramer, S. C., and Cohen, M. J. (2005). Brain activation during execution and motor imagery of novel and skilled sequential hand movements. Neuroimage 27, 505-519.

Lacourse, M. G., Turner, J. A., Randolph-Orr, E., Schandler, S. L., and Cohen, M. J (2004). Cerebral and cerebellar sensorimotor plasticity following motor imagery-based mental practice of a sequential movement. J. Rehabil. Res. Dev. 41, 505-523.

Luft, A. R., Skalej, M., Stefanou, A., Klose, U., and Voigt, K. (1998). Comparing motion- and imagery-related activation in the human cerebellum: a functional MRI study. Hum. Brain Mapp. 6, 105-113.

Mamassis, G., and Doganis, G. (2004). The effects of a mental training program on juniors pre-competitive anxiety, self-confidence, and tennis performance. J. Appl. Sport Psychol. 16, 118-137.

Medina, J. F., Garcia, K. S., and Mauk, M. D. (2001). A mechanism for savings in the cerebellum. J. Neurosci. 21, 4081-4089.

Meister, I. G., Krings, T., Foltys, H., Boroojerdi, B., Muller, M., Topper, R., and Thron, A. (2004). Playing piano in the mind - an fMRI study on music imagery and performance in pianists. Brain Res. Cogn. Brain Res. 19, 219-228.

Middleton, F. A., and Strick, P. L. (2000). Basal ganglia and cerebellar loops: motor and cognitive circuits. Brain Res. Rev. 31, 236-250.

Naito, E., Kochiyama, T., Kitada, R., Nakamura, S., Matsumura, M., Yonekura, Y., and Sadato, N. (2002). Internally simulated movement sensations during motor imagery activate cortical motor areas and the cerebellum. J. Neurosci. 22, 3683-3691.

Nyberg, L., Eriksson, J., Larsson, A., and Marklund, P. (2006). Learning by doing versus learning by thinking: an fMRI study of motor and mental training. Neuropsychologia 44, 711-717.

Obayashi, S. (2004). Possible mechanism for transfer of motor skill learning: implication of the cerebellum. Cerebellum 3, 204-211.

Olsson, C.-J., Jonsson, B., Larsson, A., and Nyberg, L. (2008). Motor representations and practice affect brain systems underlying imagery: an fMRI study of internal imagery in novices and active high jumpers. Open Neuroimaging J. 2, 5-13.

Penhune, V. B., and Doyon, J. (2005). Cerebellum and M1 interaction during early learning of timed motor sequences. Neuroimage 26, 801-812.

Peynircioglu, Z. F., Thompson, J. L. W., and Tanielian, T. B. (2000). Improvement strategies in free-throw shooting and grip-strength tasks. J. Gen. Psychol. 127, $145-156$.

Picard, N., and Strick, P. L. (2001). Imaging the premotor areas. Curr. Opin. Neurobiol. 11, 663-672.

Pie, J. S., and Tenenbaum, G. (1996). Imagery orientation and vividness: their effect on a motor skill performance. J. Sport Behav. 19, 32.

Rorden, C., and Brett, M. (2000). Stereotaxic display of brain lesions. Behav. Neurol. 12, 191-201.

Seidler, R. D. (2004). Multiple motor learning experiences enhance motor adaptability. J. Cogn. Neurosci. 16, 65-73.

Sergent, J., Zuck, E., Terriah, S., and MacDonald, B. (1992). Distributed neural network underlying musical sight-reading and keyboard performance. Science 257, 106-109.

Tanji, J. (2001). Sequential organization of multiple movements: involvement of cortical motor areas. Annu. Rev. Neurosci. 24, 631-651.

Thach, W. T. (2007). On the mechanism of cerebellar contributions to cognition. Cerebellum 6, 163-167.

Wohldmann, E. L., Healy, A. F., and Bourne, L. E. (2007). Pushing the limits of imagination: mental practice for learning sequences. J. Exp. Psychol. Learn. Mem. Cogn. 33, 254-261.

Wolpert, D. M., Ghahramani, Z., and Jordan, M. I. (1995). An internal model for sensorimotor integration. Science 269, 1880-1882.

Yousry, T. A., Schmid, U. D., Alkadhi, H., Schmidt, D., Peraud, A., Buettner, A., and Winkler, P. (1997). Localization of the motor hand area to a knob on the precentral gyrus. A new landmark. Brain 120, 141-157. 